\title{
Effect of biotransformation on patchouli oil using GC-MS
}

\author{
Navneet Khare, Dharmendra Khokhar, Soumitra Tiwari, Yashwant Kumar and Yogesh Kumar
}

Received : 10.04.2018; Accepted : 13.04.2018

See end of the Paper for authors' affiliation

Correspondence to :

\section{Navneet Khare} Department of Agricultural Processing and Food Engineerng, S.V. College of Agricultural Engineering and Technology (Indira Gandhi Krishi Vishwavidyalaya), Raipur (C.G.) India Email : navneet91001@gmail. com
- Abstract : Biotransformation is the chemical modification made by a microorganism on small molecules as well as on macro-molecules of biological origin. This process results in increased patchouli alcohol content along with improvement in oil extraction. A series of experiments were carried out to understand the biotransformation effect of three selected microorganisms on the quantity and quality of patchouli oil. Dry herbage was the substrate treated with the microbial inoculants. Higher end analysis of the oil samples with GC-MS indicated the effect biotransformation efficiency of different microorganisms on the patchouli oil component. Patchouli alcohol, the active component of the oil is found $31.78,33.73$ and $35.56 \%$, for the oils extracted after the incubation with Aspergillus foetidus, Penicillium citrinum, Trichosporon asteroides, respectively. While the oil extracted from the fresh and control samples contain 26.63 and $27.35 \%$ patchouli alcohol. Other components of the oil also affected by the fermentation/biotransformation process. From the above it can be suggested that fermentation/ bitransformation of patchouli is important for the oil recovery as well as patchouli alcohol percentage.

- Key words : Biotransformation, Patchouli oil, Patchouli alcohol, Fungi

- How to cite this paper : Khare, Navneet, Khokhar, Dharmendra, Tiwari, Soumitra, Kumar, Yashwant and Kumar, Yogesh (2018). Effect of biotransformation on patchouli oil using GC-MS. Internat. J. Agric. Engg., 11(Sp. Issue) : 53-64, DOI: 10.15740/HAS/IJAE/11.Sp. Issue/53-64. 